\title{
Hypofractionated IMRT Breast Treatment with Simultaneous Versus Sequential Boost Techniques
}

\author{
Suresh Moorthy ${ }^{1 *}$, Elhateer $\mathbf{H}^{1}$, Saroj Kumar Das Majumdar ${ }^{1}$, Shubber ${ }^{1}$, Zainab ${ }^{1}$ and Narayana Murthy $\mathbf{P}^{2}$
}

${ }^{1}$ Department of Oncology \& Hematology, Salmaniya Medical Complex, Kingdom of Bahrain

${ }^{2}$ Department of Physics, Acharya Nagarjuna University, Guntur, India

\begin{abstract}
Purpose: Whole breast irradiation is part of breast conservative management for early breast cancer; addition of boost dose to tumor bed improves local recurrence rates and is currently the standard of care. Randomized trials reported low a/b ratio for breast cancer that predict a radiobiological advantage for hypofractionation. Simultaneous boost radiation as a method of hypofractionation proved safe and effective for head and neck tumors. In this study we attempt to compare and analyze the dosimetric aspects of adding Simultaneous Integrated Boost (SIB) over Sequential Boost (SB) to a hypofractionated treatment schedule in breast cancer patients after BCS.

Materials and methods: CT simulation data sets for 23 patients were selected for this planning study; Targets and OAR were delineated as per RTOG guidelines. Multiple dynamic field IMRT plans were generated for each patient. The prescribed dose was $40 \mathrm{~Gy} / 15$ fractions to whole breast $(2.67 \mathrm{~Gy} /$ fraction) and $48 \mathrm{~Gy} / 15$ fractions to lumpectomy cavity (3.2 Gy/fraction) for SIB, and $40 \mathrm{~Gy} / 15$ fractions followed by $10 \mathrm{~Gy} / 5$ fractions for SB. Generated Treatment plans were evaluated by experienced radiation oncologist, and the best plan was selected for the dosimetric analysis.

Results: The pre specified target coverage criteria were met for the lumpectomy cavity as well as whole breast in all plans. All quality indices for PTV coverage showed to be significantly improved with SIB for both whole breast and tumor bed volumes. SB technique showed more dose spillage outside the boost volume. SIB-IMRT was better in sparing OAR, the volume of the ipsilateral lung V20 Gy was $19.8 \%$ compared to $22.8 \%(p=0.04)$, maximum dose to LAD was 17.6 Gy Vs. $21.6(p=0.01)$ and contralateral breast mean dose was 0.36 Gy Vs. 1.27 Gy $(p=0.01)$ for SIB and SB respectively.
\end{abstract}

Conclusions: Hypofractionated breast SIB is feasible with better PTV coverage and OAR. Along with further reduction of the overall period which may increase patient convenience and resource utilization benefit.

Keywords: Hypofractionation; Simultaneous Integrated Boost (SIB); Intensity Modulated Radiotherapy (IMRT); Sequential Boost (SB); Left Anterior Descending Artery (LAD)

\section{Introduction}

Breast cancer is the leading cause of cancer related deaths in women worldwide [1]. The main treatment is surgery, conservative breast surgery (BCS) followed by radiotherapy offers equivalent survival as modified radical mastectomy with better cosmetic outcomes. Whole breast Radiation therapy is the current standard of care after BCS. Tumor bed boost was associated with improved local recurrence rate $6.2 \%$ compared to $10.2 \%$ without boost $[2,3]$.

Adjuvant radiotherapy significantly improves the overall survival of breast cancer patients. This result can be augmented with further decreasing the dose to normal tissues such as heart, lung and use of IMRT and use of optimal fractionation schedules. Van de Steene et al. reported that under optimal conditions the odds of death reduction and observed 10 year mortality reduction due to adjuvant radiotherapy can be expected to be at least $20 \%$ [4].

Multiple randomized studies reported low $\mathrm{a} / \mathrm{b}$ value in the range of 3-4 for breast cancer that predict a potential radiobiological advantage for hypofractionated radiotherapy, furthermore, it can improve patient's convenience and utilization health care facilities $[5,6]$. The investigators from Standardization of Breast Radiotherapy (START) trials assessed local control rate and patient's quality of life after hypofractionated radiotherapy, there was no significant difference in loco-regional control, mild and marked breast appearance in patients who received $50 \mathrm{~Gy}$ in 25 fractions versus 41.6 in 13 fractions Gy [5].
Introduction of Intensity Modulated Radiotherapy (IMRT) to breast cancer treatment further improved conformity and doses to normal tissue. IMRT not only improves dose homogeneity but might reduce the dose to the normal structures compared to conventional wedged fields $[7,8]$.

Total treatment period can be further reduced with the use of SIB that proved safe and effective in other disease sites like head and neck, lung and prostate. In breast there are increasing clinical evidences suggesting similar advantages of SIB [9-13].

The objective of this study is to analyze the quality of PTV coverage and doses to the organs at risk (OAR) associated with hypofractionated whole breast irradiation with simultaneous integrated boost (SIB) compared to sequential boost (SB) using dynamic field IMRT technique.

*Corresponding author: Suresh Moorthy, M.Sc, M.Phil, Senior Medical Physicist, Division of Radiation Oncology, Department of Oncology \& Hematology, Salmaniya Medical Complex, MOH, Kingdom of Bahrain, Tel:+973-33523429; E-mail: nmsureshm@yahoo.com

Received March 30, 2012; Accepted May 21, 2012; Published May 24, 2012

Citation: Moorthy S, Elhateer H, Das Majumdar SK, Shubber, Zainab, et al. (2012) Hypofractionated IMRT Breast Treatment with Simultaneous Versus Sequential Boost Techniques. J Nucl Med Radiat Ther 3:130. doi:10.4172/2155-9619.1000130

Copyright: (c) 2012 Moorthy S, et al. This is an open-access article distributed under the terms of the Creative Commons Attribution License, which permits unrestricted use, distribution, and reproduction in any medium, provided the original author and source are credited. 
Citation: Moorthy S, Elhateer H, Das Majumdar SK, Shubber, Zainab, et al. (2012) Hypofractionated IMRT Breast Treatment with Simultaneous Versus Sequential Boost Techniques. J Nucl Med Radiat Ther 3:130. doi:10.4172/2155-9619.1000130

\section{Materials and Methods}

The CT simulation data of previously treated 23 patients with early stage breast cancer T1-T2 node negative (12 left sides and 11 right sides) were used for our retrospective planning study. These patients were treated by conventional fractionation with SIB-IMRT. The same CT data sets, target volumes and Organ at risk volumes were used for this study.

\section{Simulation}

All patients were simulated using Four Dimensional Computed Tomography (4DCT) (Philips Medical Systems-6 slice CT SIM) with Whole body Vaclok (Civco Medical Solutions, USA) immobilization system. Patients were trained before simulation for Breath hold technique. Patients were positioned in wide bore CT-SIM couch with the help of lasers where both arms raised above the head. Radio opaque markers were placed during the procedure to guide the isocenter shift. CT scan images were obtained from mandible to upper abdomen area with IV contrast. CT scan slice thickness of $5 \mathrm{~mm}$ was used for planning.

Organ motion during the IMRT treatment has been accounted for using real-time position management (RPM Varian, Palo Alto, USA). The reflective markers placed at the position of the xyphoid process and respiration data registered. During treatment the system supports automatic on and off triggering of radiation beam during breath hold. The marker position approximates identical and in-phase alignment of breast and marker motion.

\section{Target delineation}

After Planning CT Scan is done, the DICOM images were transferred to Eclipse treatment planning system (version 10.0.34, Varian Medical Systems, USA). Then Planning Target Volume (PTV) and Organ at Risk Volumes were delineated. The lumpectomy gross tumor volume (L-GTV) was contoured using all available clinical and radiographic information including the excision cavity volume, architectural distortion, lumpectomy scar, seroma and/or extent of surgical clips. CTV2 was created by $1 \mathrm{~cm} 3 \mathrm{D}$ expansion L-GTV and was limited posteriorly at anterior surface of the pectoralis major and antero-laterally $3 \mathrm{~mm}$ from skin, PTV2 was created by $7 \mathrm{~mm} \mathrm{3D}$ expansion of CTV2.

CTV 1 included the palpable breast tissue demarcated with radioopaque markers at CT simulation. The apparent CT glandular breast tissue visualized by CT, consensus definitions of anatomical borders, and the Lumpectomy CTV from the RTOG breast cancer atlas. The breast CTV is limited anteriorly within $3 \mathrm{~mm}$ from the skin and posteriorly to the anterior surface of the pectoralis, serratous anterior muscle excluding chest wall. Breast PTV (PTV1) was created by 3D expansion of CTV 1 by $7 \mathrm{~mm}$. The normal structures were contoured as Ipsilateral Lung, Contra lateral Lung, Contra lateral Breast, Heart, LAD (Left Anterior Descending Artery), Spinal cord, Esophagus, Trachea, humerus head and Liver.

\section{Planning details and dose prescription}

Multiple IMRT plans were created for Clinac 600CD Linear Accelerator (Varian Medical Systems, USA) which is integrated with 120 leaves Millennium MLC. Treatment fields were almost evenly spaced within an arc of 180 degree on the side of the tumor. Gantry angles ranged from 330 to 150 (clockwise) for left side tumors and from 50 to 200 (counterclockwise) for right side tumors.
All IMRT plans were created in Eclipse with inverse plan optimization algorithm DVO (Dose Volume Optimizer Version 10.0.28). For the dose calculation PBC (Pencil Beam Convolution Version 10.0.28) algorithm was used and leaf motions were calculated with LMC (Leaf Motion Calculator Version 10.0.28) algorithm.

Using Beams Eye View (BEV) fields were set up to minimize the dose to heart, lung, contralateral breast and maximize the dose to target coverage. Two plans were created for Sequential plan. In plan 1, multiple coplanar beams were used to produce adequate dose coverage PTV1 and PTV2. New volume (PTV1-PTV2) is created by the name whole breast excluding boost volume by subtracting boost volume with $5 \mathrm{~mm}$ margin from whole breast volume. The excess dose spillages into breast volume and coverage effectiveness were analyzed using this volume.

The dose - volume constraints to the target and critical organs were mentioned in Table 1 . Heterogeneity correction was done using Modified Batho method in Eclipse. Dose-Volume Histograms (DVH) was used to analyze the volume receiving $20 \mathrm{~Gy}, 30 \mathrm{~Gy}$ and $40 \mathrm{~Gy}$, Mean, Maximum and Minimum doses.

The dose prescribed for SIB method was 40 Gy in 15 fractions (2.67 Gy/fraction) for PTV1 and 48 Gy in 15 fractions (3.2 Gy/fraction) for PTV2 in 5 fractions per week schedule. For SB method the dose prescribed was $40 \mathrm{~Gy}$ in 15 fractions $(2.67 \mathrm{~Gy} /$ fraction) for PTV1 and 50 Gy in 20 fractions (2.5 Gy/fraction) forPTV2 in 5 fractions per week schedule.

The target dose uniformity and conformity are calculated and evaluated based on ICRU 83

The conformity index (CI) as defined in ICRU is

$\mathrm{CI}_{\text {(ref) }}=$ Volume covered by the reference dose / Volume of PTV

$\mathrm{CI}=1.0$ is ideal value

The Homogeneity Index (HI) as defined in ICRU is

$\mathrm{HI}=\left(\mathrm{D}_{2 \%}-\mathrm{D}_{98 \%} / \mathrm{D}_{50 \%}\right.$

$\mathrm{HI}=0$ (Zero) is ideal value

\begin{tabular}{lccc}
\hline Organ & Type & Target (Gy) & Volume (\%) \\
\hline PTV1-PTV2 & V95 & 38 & 95 \\
(Whole breast excluding & V110 & 44 & 20 \\
Tumor bed) & V95 & 45.6 & 95 \\
PTV2 & V110 & 52.8 & 10 \\
(Tumor bed only) & V20 & 20 & 20 \\
Ipsilateral Lung & V10 & 10 & 40 \\
& V5 & 5 & 10 \\
Contralateral Lung & MEAN & $<15$ & - \\
Heart & V25 & 25 & $<5$ \\
& & & - \\
Contralateral Breast & MEAN & $\leq 1$ & - \\
LAD & MAX & 20 &
\end{tabular}

Table 1: Optimization objectives for Hypo fractionated IMRT plans. 
Citation: Moorthy S, Elhateer H, Das Majumdar SK, Shubber, Zainab, et al. (2012) Hypofractionated IMRT Breast Treatment with Simultaneous Versus Sequential Boost Techniques. J Nucl Med Radiat Ther 3:130. doi:10.4172/2155-9619.1000130

Where

$\mathrm{D}_{2 \%}, \mathrm{D}_{98 \%}, \mathrm{D}_{50 \%}$ is dose received by $2 \%, 98 \%, 50 \%$ volume

\section{Statistical analysis}

Statistical analysis was performed using Wilcoxon Signed Rank test. This matched pair $t$ test was applied to determine the statistical difference between the doses -volume data. Values were reported in ranges. The reported $\mathrm{p}$ value is two tailed and $\mathrm{p}$ values of $<0.05$ are considered significant.

\section{Results}

The planning objectives were met in all cases with both techniques. Dose-Volume Histograms (DVH) describing the dose volume relationship of the Target as well as Normal tissues of the both techniques is presented in Table 2. DVH shows normal tissue sparing was better with SIB than SB.

\section{Breast volume}

Mean Breast volume was $1498.6 \mathrm{~cm}^{3}$ (range $913.5 \mathrm{~cm}^{3}$ to 2463.1 $\mathrm{cm}^{3}$ ). The normalized target coverage is presented in Table $2 \mathrm{a}$. The coverage of PTV 1 was significantly improved in the SIB arm $98.22 \%$ of the prescribed dose compared to $96.8 \%$ in SB arm ( $\mathrm{p}<0.01$ ).

\section{Boost volume}

Mean PTV2 volume was $190.3 \mathrm{~cm}^{3}$ (range $51.4 \mathrm{~cm}^{3}$ to $327.8 \mathrm{~cm}^{3}$ ). The normalized target coverage is presented in Table $2 \mathrm{~b}$. As for PTV1, SIB was associated with higher PTV 2 coverage $99.38 \%$ versus $97.3 \%$ $\mathrm{SB}(\mathrm{p}=0.01)$.

\section{Dose homogeneity and conformity}

There was a consistent improvement in conformity index for SIB for both PTV1 and PTV2. The conformity index for the boost dose was (0.97 for SIB Vs.0.94 SB) with resulting decreased spillage of the

\begin{tabular}{|l|l|l|l|}
\hline Dosimetric Parameter & SIB & SB & P Value \\
\hline Minimum Dose (Gy) & 25.71 & 23.81 & 0.29 \\
\hline Maximum Dose (Gy) & 45.48 & 49.87 & $<0.01$ \\
\hline Mean Dose (Gy) & 37.77 & 36.63 & 0.36 \\
\hline V95 (\%) & 98.22 & 96.87 & $<0.01$ \\
\hline V100 (\%) & 95.87 & 92.05 & 0.02 \\
\hline V110 (\%) & 18.97 & 35.12 & $<0.01$ \\
\hline Conformity Index & 0.95 & 0.92 & 0.02 \\
\hline Homogeneity Index & 0.12 & 0.18 & 0.02 \\
\hline (PTV1-PTV2) V45Gy (\%) & 13.09 & 21.71 & $<0.01$ \\
\hline
\end{tabular}

Table 2a: Comparison of Whole breast volume coverage parameter (Mean) for SIB boost and Sequential boost methods of intact breast cancer patients (Prescribed Dose 40 Gy).

\begin{tabular}{|l|l|l|l|}
\hline Dosimetric Parameter & SIB & SB & P Value \\
\hline Minimum Dose (Gy) & 37.82 & 36.57 & 0.15 \\
\hline Maximum Dose (Gy) & 53.55 & 56.08 & $<0.01$ \\
\hline Mean Dose (Gy) & 44.81 & 42.83 & 0.02 \\
\hline V95 (\%) & 99.38 & 97.3 & 0.01 \\
\hline V100 (\%) & 98.29 & 95.27 & $<0.01$ \\
\hline V110 (\%) & 5.87 & 14.78 & $<0.01$ \\
\hline Conformity Index & 0.97 & 0.94 & 0.01 \\
\hline Homogeneity Index & 0.09 & 0.14 & 0.01 \\
\hline
\end{tabular}

Table 2b: Comparison of boost volume coverage parameter (Mean) for SIB boost and Sequential boost methods of intact breast cancer patients (Prescribed Dose $48 \mathrm{~Gy})$.

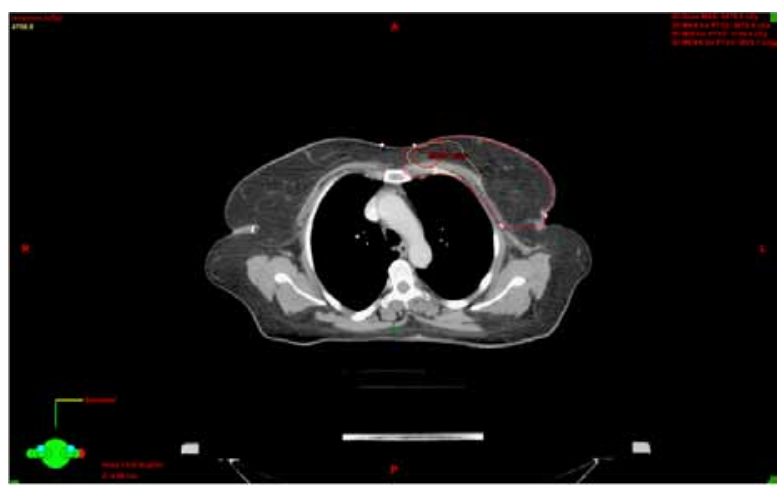

Figure 1: Sequential Breast (SB) plan sum of primary and boost which shows the spillage of dose into the breast volume from boost volume.

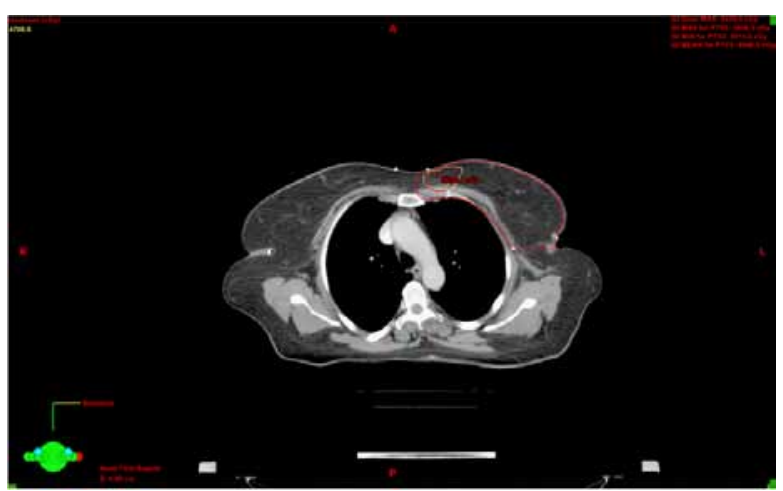

Figure 2: Simultaneous Integrated Boost (SIB) plan shows the non -spillage of dose into breast volume from boost volume.

high dose region in to surrounding breast tissue. Also the homogeneity index was significantly improved with SIB ( $\mathrm{p}=0.01)$ (Figure 1 and 2).

\section{OAR doses}

Doses to OAR showed to be significantly improved with SIB, ipsilateral lung V20 Gy and mean doses were significantly lower with SIB ( $p=0.04 \& 0.02$ ). Also, contralateral breast dose was significantly reduced with SIB $(\mathrm{p}=0.01)$. The volume of the heart receiving $30 \mathrm{~Gy}$ was reduced significantly in SIB arm (1.0\% Vs. $1.8 \%$ for SB) with $\mathrm{P}$ value of 0.01 .

SIB also was associated with significantly reduced maximum point dose to the LAD (17.59 Gy Vs. 21.61 Gy) with p value of 0.01. OAR results are presented in the Table 3 .

\section{Discussions}

The concept hypofractionated radiation therapy for breast cancer has been addressed in multiple clinical trials for its potential radiobiologic advantages as breast cancer was shown to have low $a / b$ ratio. Studies have confirmed that hypofractionated radiotherapy as effective as conventional fractionation schedules using 1.8-2 Gy per fraction with no significant compromise of the cosmetic outcomes $[5,6,14,15]$. This approach could be advantageous for patients at higher risk of local recurrence [16].

START- B trial reported no significant difference in cosmetic outcome after whole breast radiation dose of $40 \mathrm{~Gy} / 15$ fractions 


\begin{tabular}{|l|l|l|l|}
\hline Parameter & SIB & SB & P Value \\
\hline Ipsilateral Lung & & & \\
\hline V20 Gy (\%) & 19.81 & 22.79 & 0.04 \\
\hline Mean (Gy) & 12.91 & 13.26 & 0.02 \\
\hline Heart & & & \\
\hline V30 Gy (\%) & 1.0 & 1.8 & 0.01 \\
\hline V25 Gy (\%) & 3.70 & 4.11 & 0.42 \\
\hline Mean (Gy) & 9.36 & 9.57 & 0.21 \\
\hline Opposite Lung & & & \\
\hline V 5 Gy (\%) & 5.98 & 8.85 & 0.76 \\
\hline Both Lung & & & \\
\hline V20 Gy (\%) & 10.8 & 11.4 & 0.32 \\
\hline Mean (Gy) & 7.35 & 7.7 & 0.06 \\
\hline Contralateral Breast & & & \\
\hline V5 Gy (\%) & 0.84 & 3.69 & 0.06 \\
\hline Mean (Gy) & 0.36 & 1.27 & 0.01 \\
\hline LAD & & & \\
\hline Maximum Dose (Gy) & 17.59 & 21.61 & 0.01 \\
\hline
\end{tabular}

Table 3: Comparison of Mean values of Normal tissue dose volume parameters for SIB boost and Sequential boost.

compared to conventional fractionation $50 \mathrm{~Gy} / 25$ fractions based on photographic and self assessment evaluation with similar local regional tumor control rates [6]. In START A trail the late effects were significantly less with $39 \mathrm{~Gy}$ in 13 fractions over 5 weeks compared to standard fractionation $50 \mathrm{~Gy} / 25$ fractions with no significant difference in local control rate. Moreover long term result of a Canadian trial on Hypofractionated radiotherapy for breast cancer has confirmed that hypofractionated whole-breast irradiation was not inferior to standard radiation treatment in terms of tumor control as well as cosmetic outcome [17].

All the above mentioned studies were conducted prior to adoption of IMRT for treatment of breast cancer; IMRT might further decrease the risk of side-effects, consequently favoring the use of hypofractionated regimens. In fact, IMRT, due to improved conformity and homogeneity of dose distribution, reduces the dose to the OAR and it has been shown to reduce rates of acute radiation dermatitis during WBI. Currently, IMRT is in widespread use by many centers worldwide [18].

Hypofractionated WBI schedule for SB lasts 4 week's duration inclusive of boost radiation. In contrast SIB schedule delivers boost dose along with whole breast simultaneously, reduces the total treatment duration by 1 week. The radiobiologic data asserts that the shorter the overall period of treatment (especially for low $\mathrm{a} / \mathrm{b}$ tumors) good tumor control probability. Also shorter overall treatment period would be more convenient for patients and for health care providers, as it would increase the utilizing radiation therapy facility.

In an analysis of late effects in patients treated in the UK FAST trial on hypofractionated whole breast radiotherapy with 3DCRT; Goldsmith et al reported that large breast size is associated with high risk for adverse effect; the mean breast volume in that trial was 1357 cc [19], in our study, the mean breast volume was $1498 \mathrm{cc}$. The use of IMRT allowed us to achieve adequate PTV coverage in both arms, with acceptable quality; SIB was associated with significantly improved dose homogeneity and dose to OAR over the SB. Also dose conformity measured by the RTOG CI was significantly improved with SIB that resulted in marked decrease of the boost dose spillage into the surrounding breast tissue.

Multiple dosimetric studies have reported improved homogeneity

of dose distribution with the use of IMRT which result in reduced dose to the heart and lungs, and contralateral breast [20-23]. A feasibility study on the use of Simultaneous Boost Radiotherapy with IMRT improved boost coverage and decreased OAR doses, compared with sequential boost [18]. Our institutional dose volume constraint for the lung in breast radiotherapy is to keep V20 at $<30 \%$ of the ipsilateral lung using conventional fractionation as the risk of radiation pneumonitis shown to be increased with increased both lungs V20 above 30\% [24] For the current study we have tried to keep V20 at $20 \%$ with the lowest possible mean lung dose (MLD). SIB arm showed statistically significant improvement of the MLD as well as the ipsilateral lung V 20 Gy with an average of $12.9 \mathrm{~Gy}(\mathrm{p}=0.04)$ and $19.8 \%(\mathrm{p}=0.02)$ respectively. These results are in line with the results from Chen et al who reported average ipsilateral lung V20 of $20.9 \%$ using the same dose schedule [25].

Dose to heart and LAD was also reduced significantly with the use of SIB, the mean V30 Gy was $1 \%$ compared to $1.8 \%$ for $\mathrm{SB}(\mathrm{p}=0.01$ ), the maximum point dose to LAD was 17.6 with SIB versus 21.6 for SB $(p=0.01)$. Heart dose higher than $30 \mathrm{~Gy}$ is associated with increased incidence of coronary arterial disease (CAD) [26]. The advances in the treatment techniques including IMRT reduced cardiac exposure so that steady decline of Radiation Risk is being noticed [27]

Overall, SIB seems to be superior in terms of dosimetry planning. This optimized result could be attributed to optimization of inverse planning done for both primary as well as boost plans simultaneously. Since single plan, we had better control of optimization techniques than sum of optimized plans as in SB. Also SB planning and treatment is time consuming than SIB. As on today, limited studies are available for breast hypofractionation with SIB IMRT for further comparison.

\section{Conclusion}

This feasibility study of concomitant boost radiotherapy using IMRT demonstrates excellent dosimetric coverage of the boost volume, with better sparing of normal tissue, compared to Sequential Boost Technique. The overall treatment course was reduced by 1 week which may lead to patient convenience. Clinical studies will conclude the long term toxicity and efficacy.

\section{References}

1. Kamangar F, Dores GM, Anderson WE (2006) Patterns of cancer incidence mortality, and prevalence across five continents: defining priorities to reduce caner disparities in different geographic regions of the world. J Clin Oncol 24 2137-2150.

2. Bartelink H, Horiot JC, Poortmans PM, Struikmans H, Van den Bogaert W, et al (2007) Impact of a higher radiation dose on local control and survival in breastconserving therapy of early breast cancer: 10-year results of the randomized boost versus no boost EORTC 22881-10882 trial. J Clin Oncol 25: 3259-3265.

3. Collette S, Collette L, Budiharto T, Horiot JC, Poortmans PM, et al. (2008) Predictors of the risk of fibrosis at 10 years after breast conserving therapy for early breast cancer: a study based on the EORTC Trial 22881-10882 'boost versus no boost'. Eur J Cancer 44: 2587-2599.

4. Van de Steene J, Soete G, Storme G (2000) Adjuvant radiotherapy for breast cancer significantly improves overall survival: the missing link. Radiother Oncol 55: 263-272

5. START Trialists' Group, Bentzen SM, Agrawal RK, Aird EG, Barrett JM, et al. (2008) The UK Standardisation of Breast Radiotherapy (START) Trial A of radiotherapy hypofractionation for treatment of early breast cancer: a randomised trial. Lancet Oncol 9: 331-341.

6. Yarnold J, Ashton A, Bliss J, Homewood J, Harper C, et al. (2005) Fractionation sensitivity and dose response of late adverse effects in the breast afte radiotherapy for early breast cancer: long-term results of a randomised trial. Radiother Oncol 75: 9-17.

7. Borghero YO, Salehpour M, McNeese MD, Stovall M, Smith SA, et al. (2007) 
Citation: Moorthy S, Elhateer H, Das Majumdar SK, Shubber, Zainab, et al. (2012) Hypofractionated IMRT Breast Treatment with Simultaneous Versus Sequential Boost Techniques. J Nucl Med Radiat Ther 3:130. doi:10.4172/2155-9619.1000130

Multileaf field-in-field forward-planned intensity-modulated dose compensation for whole-breast irradiation is associated with reduced contralateral breas dose: a phantom model comparison. Radiother Oncol 82: 324-328.

8. Nieder C, Schill S, Kneschaurek P, Molls M (2007) Influence of different treatment techniques on radiation dose to the $L A D$ coronary artery. Radiat Oncol 2: 20

9. Coen W. Hurkmans, Gert J. Meijer, Corine van Vliet-Vroegindeweij, Maurice $\mathrm{J}$. van der Sangen, Jorien Cassee (2006) High dose simultaneously integrated breast boost using intensity modulated radiotherapy and inverse optimisation. Int J Radiat Oncol Biol Phys 66: 923-930.

10. Singla R, King S, Albuquerque K, Creech S, Dogan N (2006) Simultaneous integrated boost intensity modulated radiation therapy (SIB-IMRT) in the treatment of early stage left sided breast carcinoma. Med Dosim 31:190-196.

11. van der Laan HP, Dolsma WV, Maduro JH, Korevaar EW, Hollander M, et al. (2007) Three.dimensional conformal simultaneously integrated boost technique for breast conserving radiotherapy. Int J Radiat Oncol Biol Phys 68:1018-1023.

12. McDonald MW, Godette KD, Whitaker DJ, Davis LW, Johnstone PA (2010) Three-year outcomes of breast intensity-modulated radiation therapy with simultaneous integrated boost. Int J Radiat Oncol Biol Phys 77: 523-530.

13. Hijal T, Fournier-Bidoz N, Castro-Pena P, Kirova YM, Zefkili S, et al. (2010) Simultaneous integrated boost in breast conserving treatment of breast cancer: a dosimetric comparison of helical tomotherapy and three-dimensional conformal radiotherapy. Radiother oncol 94: 300-306

14. START Trialists' Group, Bentzen SM, Agrawal RK, Aird EG, Barrett JM, et al. (2008) The UK Standardisation of Breast Radiotherapy (START) Trial B of radiotherapy hypofractionation for treatment of early breast cancer: a randomised trial. Lancet 371: 1098-1107.

15. Qi XS, White J, Li XA (2011) Is alpha/beta for breast cancer really low? Radiother Oncol 100: 282-288.

16. Antonini N, Jones H, Horiot JC, Poortmans $\mathrm{P}$, Struikmans $\mathrm{H}$, et al. (2007) Effect of age and radiation dose on local control after breast conserving treatment: EORTC trial 22881-10882. Radiother Oncol 82: 265-271.
17. Whelan TJ, Pignol JP, Levine MN, Julian JA, MacKenzie R, et al. (2010) Longterm results of hypofractionated radiation therapy for breast cancer. $\mathrm{N}$ Engl J Med 362: 513-520.

18. Teh AY, Walsh L, Purdie TG, Mosseri A, Xu W, et al. (2012) Concomitant intensity modulated boost during whole breast hypofractionated radiotherapy-a feasibility and toxicity study. Radiother Oncol 102: 89-95.

19. Goldsmith C, Haviland J, Tsang Y, Sydenham M, Yarnold J, et al. (2011) Large breast size as a risk factor for late adverse effects of breast radiotherapy: is residual dose inhomogeneity, despite $3 \mathrm{D}$ treatment planning and delivery, the main explanation? Radiother Oncol 100: 236-240.

20. Woo TC, Pignol JP, Rakovitch E, Vu T, Hicks D, et al. (2006) Body radiation exposure in breast cancer radiotherapy: impact of breast IMRT and virtual wedge compensation techniques. Int J Radiat Oncol Biol Phys 65: 52-58.

21. Thilmann C, Zabel A, Nill S, Rhein B, Hoess A, et al. (2002) Intensity-modulated radiotherapy of the female breast. Med Dosim 27: 79-90.

22. Landau D, Adams EJ, Webb S, Ross G (2001) Cardiac avoidance in breas radiotherapy: a comparison of simple shielding techniques with intensitymodulated radiotherapy. Radiother Oncol 60: 247-255.

23. Rong Y, Fahner T, Welsh JS (2008)Hypofractionated breast and chest wal irradiation using simultaneous in-field boost IMRT delivered via helical tomotherapy. Technol Cancer Res Treat 7: 433-439.

24. Graham MV, Purdy JA, Emami B, Harms W, Bosch W, et al. (1999) Clinical dose volume histogram analysis for pneumonitis after 3D treatment for nonsmall cell lung cancer (NSCLC). Int J Radiat Oncol Biol Phys 45: 323-329.

25. Chen G, White J, Vicini FA, Freedman GM, Li A (2011) dosimetric planning study for hypofractionated Whole breast irradiation with concurrent boost (RTOG 1005) for early stage breast cancer. Proceeding of the ASTRO.

26. Gagliardi G, Constine LS, Moiseenko V, Correa C, Pierce LJ, et al. (2010) Radiation dose-volume effects in the heart. Int J Radiat Oncol Biol Phys 76 S77-85

27. Giordano SH, Kuo YF, Freeman JL, Buchholz TA, Hortobagyi GN, et al. (2005) Risk of cardiac death after adjuvant radiotherapy for breast cancer. J Nat Cancer Inst 97: 419-424. 